\title{
Final attack ratio in SIR epidemic models for multigroup populations
}

\author{
Antonella Lunelli · Andrea Pugliese
}

Received: date / Accepted: date

\begin{abstract}
We analyse an SIR epidemic model in a closed population subdivided in $n$ groups. Population mixing occurs at two levels: within each group, and uniformly in the population. We prove that, if within-group transmission rates are large enough and not all identical to each other, then the final attack ratio is lower than what would occur in a population mixing homogeneously with the average transmission rate. We also show that the opposite may hold for certain parameter values and explore numerically the parameter regions in which the final attack ratio is higher or lower than in the corresponding homogeneous model. Finally, we analyse simulations of the corresponding stochastic model with finite group size, studying how well final attack ratio is approximated by the deterministic outcome and its relations with exponential growth rate.
\end{abstract}

Keywords Epidemic metapopulation model · Epidemic attack ratio · Epidemic model with two levels of mixing

Mathematics Subject Classification (2010) MSC 92D30

\section{Introduction}

The progress of epidemic modelling has stressed the importance, among other features, of the structure of contacts in the population, going beyond the simple homogeneous mixing [30]. A very popular approach, helped by the advancement of computing power, has been the use of agent-based models based on a synthetic population, in which individual contacts are kept track of $[19,24,35]$. While this class of

\footnotetext{
A. Lunelli

Dip. di Matematica, Università di Trento

E-mail: antonella.lunelli@gmail.com

A. Pugliese

Dip. di Matematica, Università di Trento

E-mail: andrea.pugliese@unitn.it
} 
models makes it possible to reach a very satisfying description of actual epidemics, to perform scenario analysis and predict the impact of control measures, they are hardly amenable to theoretical analysis.

A different approach, aiming at obtaining theoretical insights on the effect of contact structures on epidemic dynamics, has been to consider simple models in which the population is partitioned into groups [6]. Some models of this type are deterministic, starting from the work by Hethcote and Yorke on gonorrhea [25] and generalised in many different ways, modelling social groups, as in [25,34], or spatial spread [41, 28,23 ], in which case they are often called metapopulation models. Other models are stochastic, where often the groups being modelled represent households [7], while more recently the presence of different and intertwining mixing groups (for instance, households and workplaces) has been allowed [9]. Here, we restrict the analysis to models of a single epidemic, in which there is no replacement of susceptibles; results on stochastic models for the endemic case are much more scarce [6].

A general question that can be asked in this type of models concerns the effect of heterogeneities. It was proved, in the context of HIV/AIDS modelling with proportional mixing, that heterogeneities in contact rates increase the reproduction number $R_{0}$ relatively to the corresponding model in which each group has the average contact rate [34], and thus make an epidemic outbreak easier. On the other hand, it has been proved by Andreasen [3] that, with proportional mixing, heterogeneities in contact rates decrease the final attack ratio relatively to the corresponding model with homogeneous contact rates and the same value of $R_{0}$. The final attack ratio is the proportion of the population that gets infected by the end of the epidemic, a quantity that is also named 'epidemic final size', 'epidemic impact' or 'attack rate'; we will use here the term 'final attack ratio', as it is a ratio and not a rate.

Such conclusions appear intuitive, but, as far as we know, have not been established in more general contexts. Here, we analyse the conditions under which heterogeneity in contact rates decreases the epidemic impact in a class of deterministic metapopulation models for an SIR epidemic. The models are similar to the multigroup models with preferred mixing introduced by Nold [37] and extend previous results [3] in a different direction. The idealized situations to which the model could apply are different towns and villages within a region, or different schools and workplaces within a city. Especially in the latter case, it is reasonable to expect that young children in a kindergarten will have a very different mixing behaviour from teenagers in a high school or workers in an office or a factory, and thus that within-groups contact rates may vary between each other.

It follows from well-known limit theorems [18] that stochastic metapopulation models will converge to deterministic systems, as the size of each group tends to infinity. Through stochastic simulations, we investigate the group size around which the attack ratio predicted by the deterministic model becomes a good approximation of the outcome of the stochastic model, at least for simulations that avoid early extinctions.

The analysis of stochastic simulations lets us explore also whether the final attack ratio can be reasonably predicted from measurements performed at the beginning of an epidemic, in particular from the exponential growth rate, parameter that is often estimated in the early phase of an epidemic [36,31]. Indeed, in epidemic models 
for a homogeneously mixing populations, both final attack ratio $z$ and exponential growth rate $r$ are (as long as recovery rate $\gamma$ is kept fixed) increasing functions of the reproduction number $R_{0}$, so that from an estimate of $r$ one can infer an estimate of $z$. One may wonder whether a relationship, perhaps somewhat different from the one holding in the homogeneous case, can also be found for simple metapopulation models.

\section{The SIR meta-population model}

We consider an epidemic model with an SIR structure, in which the individuals are classified according to their disease state, as susceptible (S), infectious (I) or recovered (R). Heterogeneity is introduced by subdividing the population in $n$ groups representing geographic regions or social structures (e.g., schools and workplaces). Transmission may occur through a contact with an infectious individual of the same group or of a different group. In other words, we have two levels of mixing, one local between individuals of the same group $i$, regulated by a transmission rate $\tilde{\beta}_{i}$ $(i=1, \ldots n)$, and one global, between individuals of different groups, regulated by the transmission coefficient $\beta_{g}$, constant and independent of the groups involved.

The model is described by the following set of differential equations:

$$
\left\{\begin{array}{l}
\dot{S}_{i}=-S_{i}\left(\beta_{i} \frac{I_{i}}{N_{i}}+\lambda_{g}\right) \\
\dot{I}_{i}=S_{i}\left(\beta_{i} \frac{I_{i}}{N_{i}}+\lambda_{g}\right)-\gamma I_{i} \\
\dot{R}_{i}=\gamma I_{i}
\end{array}\right.
$$

with

$$
\lambda_{g}=\frac{\beta_{g}}{n} \sum_{j=1}^{n} \frac{I_{j}}{N_{j}} .
$$

In system (1), $N_{i}$ represents the number of individuals in group $i$, and it is assumed that global contacts are taken at the same rate with all groups. From (1) and (2), one may note that the contact rate within group $i$ is actually equal to $\tilde{\beta}_{i}=\beta_{i}+\beta_{g} / n$; the use of $\beta_{i}$, instead of $\tilde{\beta}_{i}$ makes the notation simpler.

An alternative assumption for global contacts is to assume that such contacts are proportional to the size of each group, i.e.

$$
\lambda_{g}=\frac{\beta_{g} \sum_{j=1}^{n} I_{j}}{\sum_{j=1}^{n} N_{j}} .
$$

Most of the following analysis will be performed using expression (2) for $\lambda_{g}$; results with (3) are qualitatively the same, but there are some differences in the computations, that will be briefly sketched. 
Finally, $\gamma$ is the rate at which individuals recover. By changing the time unit, we can set $\gamma=1$, as will always be assumed in what follows [3].

This model is very similar (but not identical, because here $\beta_{g}$ is assumed to be the same for all groups, while $\beta_{i}$ may differ) to the preferred mixing model first proposed by Nold [37], and generalized and analysed by several authors [25, 12,4].

This structure of infection transmission has been used many times in stochastic setting [7], initially assuming that groups correspond to households, but then generalizing to more complex structures (see for instance $[9,38,8]$ ). In these models, groups are generally assumed to be small, so that the dynamics of within-group epidemics have a strongly stochastic component, which makes deterministic models such as (1) not appropriate (different deterministic models may be appropriate [26], but they are difficult to analyse).

On the other hand, time to extinction in a stochastic version of model (1) has been studied by Hagenaars et al. [23] and Lindholm and Britton [32], using approximations valid for somewhat larger groups, thus modelling a setting similar to the one analysed here.

\subsection{The basic reproduction number}

The basic reproductive number provides an aggregated measure of the transmissibility of an infection. In a simple homogeneous model, it is defined as the average number of individuals infected by a newly infected individual over the entire infectious period in a completely susceptible population and it is directly related both to the initial growth rate and to the final attack ratio (see below). Moreover it determines the threshold between an infection that can spread and one that cannot.

From a theoretical point of view, a rigorous definition is given in [17], where $R_{0}$ is defined as the dominant eigenvalue of the next generation matrix, whose $i j$ entry is the expected number of secondary cases in group $i$ caused by an infectious individual in group $j$. In the case of system (1)-(2), it can be easily seen that the elements of the next-generation matrix are

$$
K_{i j}=\beta_{i} \delta_{i j}+\frac{\beta_{g}}{n} \frac{N_{i}}{N_{j}}, \quad i, j=1 \ldots n
$$

so that $R_{0}=\rho(K) . R_{0}$ represents the threshold between an infection that can spread and one that dies out spontaneously [17], and is also related to the fraction of individuals that needs to be vaccinated in each group to stop the disease invasion.

When the internal transmission rate is the same in all groups $\left(\beta_{i} \equiv \beta\right)$, the leading eigenvalue of $K$ can be easily computed and does not depend either on the number of groups or on their dimension. Specifically, we find

$$
R_{0}=\beta+\beta_{g} .
$$

Remember that (4) has been obtained assuming a rescaling of time so that $\gamma=1$; in normal time units, one should divide (5) by $\gamma$. 
If $\beta_{i}$ are not identical, a simple computation shows that the eigenvalues of $K$ are the roots of

$$
H(\lambda):=\frac{\beta_{g}}{n} \sum_{j=1}^{n} \frac{1}{\lambda-\beta_{j}}=1 .
$$

It can be easily seen that $H$ is a decreasing, continuous function on $\left(\beta_{\max },+\infty\right)$ where $\beta_{\max }=\max _{i} \beta_{i}$ with

$$
\lim _{\lambda \rightarrow \beta_{\max }^{+}} H(\lambda)=+\infty, \quad \lim _{\lambda \rightarrow+\infty} H(\lambda)=0
$$

Hence there exists a unique real root of (6) in $\left(\beta_{\max },+\infty\right)$.

Since $K$ is a positive matrix, Perron-Frobenius theory implies that $\rho(K)$ is an eigenvalue; hence $R_{0}$ is the only root of (6) in $\left(\beta_{\max },+\infty\right)$. While it is impossible, unless $n=2$, to have an explicit expression for $R_{0}$, it is possible, as shown in $[4,17]$, to have an explicit threshold condition. Indeed, from (6) it is easy to see that $R_{0}>1$ if and only if one of the following conditions hold:

$-\max _{i} \beta_{i} \geq 1$

$-\max _{i} \beta_{i}<1$ and $H(1)=\frac{\beta_{g}}{n} \sum_{j=1}^{n} \frac{1}{1-\beta_{j}}>1$.

Furthermore, we see that

$$
H\left(\beta_{\max }+\beta_{g}\right) \leq \frac{\beta_{g}}{n} \sum_{j=1}^{n} \frac{1}{\beta_{j}+\beta_{g}-\beta_{j}}=1
$$

with a strict inequality, unless $\beta_{j}=\beta_{\max }$ for all $j$. Hence, we obtain

$$
\max _{i} \beta_{i}<R_{0}<\max _{i} \beta_{i}+\beta_{g} .
$$

This last inequality shows that the value of $R_{0}$ is high if we have high intra-group transmission, even if this occurs only in one small group and the inter-group transmission rate remains low. However, in extreme situations with high transmission only in one small group, we do not expect this to actually dominate and determine the spread of the infection. This phenomenon is explored numerically in the next Section. Note further that, when global contact rates are much smaller than within-group ones, the interval in (7) is relatively narrow, and the relative error in the simple estimate $R_{0} \approx \max _{i} \beta_{i}$ is small.

A natural question is how $R_{0}$ compares with the value (5) found when all groups have identical contact rate. Thus, we define $\bar{\beta}=\frac{1}{n} \sum_{i=1}^{n} \beta_{i}$, and show that, unless all $\beta_{i}$ are identical,

$$
R_{0}>\bar{\beta}+\beta_{g},
$$

i.e. the expression (5) found for the case when all groups have the average withingroup contact rate.

In fact, in the case (that we think would be empirically likely) that $\bar{\beta}+\beta_{g} \leq \max _{i} \beta_{i}$, this follows immediately from (7). 
If we instead assume $\bar{\beta}+\beta_{g}>\max _{i} \beta_{i}$, we can apply Jensen inequality to the function $q(x)=\frac{1}{\bar{\beta}+\beta_{g}-x}$ that is continuous and convex in $\left[0, \bar{\beta}+\beta_{g}\right)$. Then

$$
H\left(\bar{\beta}+\beta_{g}\right)=\frac{\beta_{g}}{n} \sum_{j=1}^{n} \frac{1}{\bar{\beta}+\beta_{g}-\beta_{j}}=\beta_{g} \frac{1}{n} \sum_{j=1}^{n} q\left(\beta_{j}\right)>\beta_{g} q(\bar{\beta})=\frac{\beta_{g}}{\beta_{g}}=1 .
$$

Because of the properties of the function $H(\cdot)$ in $\left(\beta_{\max },+\infty\right)$, this inequality implies (8), as required.

If (2) is substituted by expression (3) in $\lambda_{g}$, we get

$$
K_{i j}=\beta_{i} \delta_{i j}+\frac{\beta_{g} N_{i}}{\sum_{k=1}^{n} N_{k}} .
$$

It follows that $R_{0}$ is still the only root of (6) in $\left(\beta_{\max },+\infty\right)$, but with $H(\cdot)$ given by

$$
H(\lambda)=\frac{\beta_{g}}{\sum_{k=1}^{n} N_{k}} \sum_{j=1}^{n} \frac{N_{j}}{\lambda-\beta_{j}} .
$$

Still, the same conclusions, for instance (7), hold.

Also (8) still holds but with $\bar{\beta}=\frac{\sum_{i=1}^{n} N_{i} \beta_{i}}{\sum_{i=1}^{n} N_{i}}$.

2.2 The final attack ratio of the epidemic

Equations (1) represent epidemic spread in a closed population without recovery from infection, or entry of new susceptibles. Hence, the epidemic is going to finish anyway, and the interesting question is what will be the final attack ratio, i.e. the proportion of the population that eventually will have been infected.

Assuming that the epidemics has been going forever with $\lim _{t \rightarrow-\infty} S_{i}(t)=N_{i}$, the attack ratio $z_{i}$ in group $i$ is given by

$$
z_{i}=\frac{R_{i}(\infty)}{N_{i}}=\frac{1}{N_{i}} \int_{-\infty}^{+\infty} I_{i}(s) d s
$$

The final size problem for an epidemic in a subdivided population has been studied by several authors [11,5]. Recently, Andreasen [3] studied this problems assuming a general mixing patterns among subgroups. He obtained a system of equations necessarily satisfied by $z_{i}$, and showed that it has no positive solution if $R_{0}<1$ and that, under a generic assumption, it has a unique solution for $R_{0}>1$.

He further studied the problem under the assumption of proportional mixing with groups differing uniquely in susceptibility and/or infectiousness and showed that the final attack ratio is smaller in a heterogeneous population than in a homogeneous 
population with the average susceptibility or infectiousness. We study here whether the same conclusion holds under the assumption of heterogeneity in within-group contact rates, as in system (1).

Specializing equations (7) of [3] to the case of system (1), one obtains the following equations for the final attack ratios

$$
\log \left(1-z_{i}\right)+\beta_{i} z_{i}+\frac{\beta_{g}}{n} \sum_{j=1}^{n} z_{j}=0 \quad i=1 \ldots n .
$$

Indeed, these can be easily obtained by integrating (for $t$ from $-\infty$ to $+\infty) \frac{d}{d t} \log \left(S_{i}(t)\right.$ ) in (1) and using

$$
\lim _{t \rightarrow-\infty} S_{i}(t)=N_{i}, \lim _{t \rightarrow-\infty} I_{i}(t)=\lim _{t \rightarrow+\infty} I_{i}(t)=0 .
$$

The following result holds.

Theorem 1 If the internal transmission rates $\beta_{i}$ are all equal to each other, then the proportion of infections at the end of the epidemic is the same in each group. Moreover the final attack ratio of model (1) does not depend either on the number of groups, or on their dimension.

Proof If $\beta_{i} \equiv \bar{\beta}$, equations (10) do not depend on $i$; hence their solutions $z_{i} \equiv \bar{z}$. Substituting this in (10) yields

$$
G(\bar{z}):=\log (1-\bar{z})+\left(\bar{\beta}+\beta_{g}\right) \bar{z}=0 .
$$

This is the equation for the final attack ratio in the ODE version of Kermack-McKendrick model [30]. It is well known that it has a unique positive solution $\bar{z}, 0<\bar{z}<1$ if and only if $\bar{\beta}+\beta_{g}>1$; otherwise, if $\bar{\beta}+\beta_{g} \leq 1$, (11) has no positive solutions. Recalling (5), one sees that the condition for existence of positive attack ratios is $R_{0}>1$, as shown in [3] for the general case.

We found that the attack ratio is the same in each group. Consequently, the attack ratio for the whole population

$$
Z=\frac{\sum_{i=1}^{n} R_{i}(\infty)}{\sum_{i=1}^{n} N_{i}}=\frac{\bar{z} \sum_{i=1}^{n} N_{i}}{\sum_{i=1}^{n} N_{i}}=\bar{z}
$$

If expression (3) is used for $\lambda_{g}$, equation (10) is modified into

$$
\log \left(1-z_{i}\right)+\beta_{i} z_{i}+\frac{\beta_{g} \sum_{j=1}^{n} N_{j} z_{j}}{\sum_{j=1}^{n} N_{j}}=0
$$

All the conclusions of Theorem 1 hold word by word, including equation (11) for the attack ratio in each group.

In general we have 
Theorem 2 Equations (10) have a unique positive solution if and only if $R_{0}>1$. If $R_{0} \leq 1$, the only solution of (10) is $z_{i} \equiv 0$.

This result was already proved by Andreasen [3] in a more general context. We state it here as a Theorem and provide a proof because it introduces tools that are needed in the following result concerning the case where group-specific transmission rates are not all equal to each other.

Theorem 3 Assume $R_{0}>1$ and that at least one of the following conditions holds:

a) $\beta_{i}>9 / 8$ for all $i=1 \ldots n$;

b) $\beta_{i}>1$ for all $i=1 \ldots n$ and $\beta_{g}$ small enough;

c) $\beta_{g}+\beta_{\min } \geq \frac{3}{2} \log (3)$.

If the internal transmission rates $\beta_{i}$ are not all identical, then the average (among groups) attack ratio using model (1)-(2) is lower than the attack ratio obtained with $\beta_{i}$ all equal to the average value $\bar{\beta}$ and thus lower than the final attack ratio obtained with the simple homogeneous SIR model with $\beta=\bar{\beta}+\beta_{g}$.

In order to prove the Theorems, we collect some useful properties in a few lemmas.

Lemma 1 Let us define the function

$$
F(z, \beta, A)=\log (1-z)+\beta z+\beta_{g} A \quad \text { for } 0<z<1, A, \beta \geq 0 .
$$

For each $A>0, \beta \geq 0$, there exists a unique solution $z=\varphi(\beta, A)$ of $F(z, \beta, A)=0$. Furthermore, $\varphi(\beta, A)>1-\frac{1}{\beta}$.

Proof As a function of $z, F$ is a concave function that tends to $-\infty$ when $z \rightarrow 1^{-}$ and equals $\beta_{g} A$ when $z=0$. From these properties, it is easy to see that there exists a unique intersection $z=\varphi(\beta, A)$ between $F$ and the $\mathrm{x}$-axis.

Concerning the last clause, it is easy to see that, if $\beta>1$, then $F$ has a maximum in $z=1-1 / \beta$; the intersection with the $x$-axis will be to the right of this point, proving the conclusion. Otherwise, if $\beta \leq 1$, the conclusion is obvious.

For ease of reference, we rewrite the equation satisfied by $\varphi(\beta, A)$ :

$$
\log (1-\varphi(\beta, A))+\beta \varphi(\beta, A)+\beta_{g} A=0 .
$$

Lemma 2 The derivatives of $\varphi(\beta, A)$ have the following signs:

$$
\frac{\partial \varphi}{\partial A}(\beta, A)>0 \quad \frac{\partial^{2} \varphi}{\partial A^{2}}(\beta, A)<0 \quad \frac{\partial \varphi}{\partial \beta}(\beta, A)>0 .
$$

Furthermore, $\frac{\partial^{2} \varphi}{\partial \beta^{2}}(\beta, A)<0$ if one of the following conditions holds:

a') $\beta>9 / 8$;

b') $\beta>1$ and $\varphi(\beta, A)$ sufficiently close to 0 ;

c') $\varphi(\beta, A)>2 / 3$ 
Proof To prove the first property, from $F(\varphi(\beta, A), \beta, A) \equiv 0$ one has

$$
0=\frac{d}{d A} F(\varphi(\beta, A), \beta, A)=\left(-\frac{1}{1-\varphi(\beta, A)}+\beta\right) \frac{\partial \varphi}{\partial A}(\beta, A)+\beta_{g}=0 .
$$

Hence

$$
\frac{\partial \varphi}{\partial A}(\beta, A)=\frac{\beta_{g}(1-\varphi(\beta, A))}{1-\beta(1-\varphi(\beta, A))}>0
$$

given that $\varphi(\beta, A)>1-\frac{1}{\beta}$.

Differentiating (15), we have

$$
\frac{\partial^{2} \varphi}{\partial A^{2}}=-\frac{\beta_{g} \frac{\partial \varphi}{\partial A}}{(1-\beta(1-\varphi(\beta, A)))^{2}}<0 .
$$

As for the third inequality, from the implicit function theorem we have

$$
\frac{\partial \varphi}{\partial \beta}=\frac{\varphi(\beta, A)(1-\varphi(\beta, A))}{1-\beta(1-\varphi(\beta, A))}>0 .
$$

Finally, using again of the implicit function theorem, one obtains

$$
\frac{\partial^{2} \varphi}{\partial \beta^{2}}=-\frac{F_{\beta \beta}+2 F_{z \beta} \varphi_{\beta}+F_{z z}\left(\varphi_{\beta}\right)^{2}}{F_{z}} .
$$

By computing the partial derivatives of $F$ and inserting them into the previous expression, one arrives after some computations at

$$
\frac{\partial^{2} \varphi}{\partial \beta^{2}}=\frac{\frac{\partial \varphi}{\partial \beta}}{(1-\beta(1-\varphi(\beta, A)))^{2}} g(\beta, \varphi(\beta, A))
$$

with

$$
g(\beta, x)=-2(\beta-1)+(4 \beta-3) x-2 \beta x^{2} .
$$

(16) shows that $\frac{\partial^{2} \varphi}{\partial \beta^{2}}$ has the same sign as $g(\beta, \varphi(\beta, A))$. As a function of $x, g$ is a quadratic:

if $\beta>9 / 8$, its discriminant is negative, so that $g(\beta, x)$ is negative for all $x$, proving the last clause in case a').

If $1<\beta<9 / 8, g(\beta, x)$ has two roots $x_{-}$and $x_{+}$in $(0,1)$;

if $\varphi(\beta, A)<x_{-}, g(\beta, \varphi(\beta, A))<0$, proving the Lemma in case b').

For all $\beta \geq 0$, the larger root of $g(\beta, x)=0, x_{+} \leq 2 / 3$; hence, if $\varphi(\beta, A)>2 / 3$, $g(\beta, \varphi(\beta, A))<0$, proving the Lemma in case c'). 

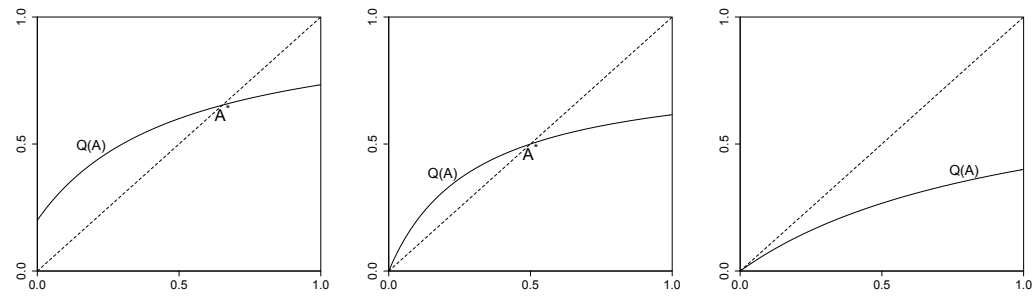

Fig. 1: The qualitative shape of the function $y=Q(A)$ (solid lines) in the three cases. Left panel: $\max _{i} \beta_{i}>1$ so that $Q(0)>0$; centre panel: $\max _{i} \beta_{i} \leq 1$ and $R_{0}>1$, so that $Q(0)=0, Q^{\prime}(0)>1$; right panel: $R_{0} \leq 1$ so that $Q(0)=0, Q^{\prime}(0) \leq 1$; the dashed lines represent the bisectrix $y=A$.

Proof (of Theorem 2) Fix $A>0$, define the function

$$
Q\left(\beta_{1} \ldots \beta_{n}, A\right)=\frac{1}{n} \sum_{j=1}^{n} \varphi\left(\beta_{j}, A\right) .
$$

A fixed point of $Q$, i.e. a solution of the equation $Q\left(\beta_{1} \ldots \beta_{n}, A\right)=A$, corresponds to a solution of (10).

Indeed, if $A$ is a fixed point of $Q$, let $z_{i}=\varphi\left(\beta_{i}, A\right)$ be the solution of the equation $F\left(z, \beta_{i}, A\right)=0$; from (17) we have $A=\frac{1}{n} \sum_{j=1}^{n} z_{j}$, while (14) yields $\log \left(1-z_{i}\right)+\beta z_{i}+$ $\beta_{g} A=0$. The two equations together are exactly (10).

Vice versa, from a solution $\left(z_{1}, \ldots, z_{n}\right)$ of (10), one defines $A=\frac{1}{n} \sum_{j=1}^{n} z_{j}$ and sees that $z_{i}=\varphi\left(\beta_{i}, A\right)$ and that $A$ is a fixed point of $Q$.

From the results of Lemma 2, one sees that, as a function of $A, Q\left(\beta_{1}, \ldots, \beta_{n}, A\right)$ is positive, increasing and concave. Furthermore $Q\left(\beta_{1}, \ldots, \beta_{n}, 1\right)<1$, since $\varphi\left(\beta_{j}, A\right)<$ 1 for all $j$ and $A$.

The behaviour of $Q$ at $A=0$ then determines whether the equation $Q\left(\beta_{1} \ldots \beta_{n}, A\right)=$ $A$ has a positive solution.

Note that $\varphi(\beta, A)$ has not been defined at $A=0$; however, one sees that

$$
\lim _{A \rightarrow 0^{+}} \varphi(\beta, A)>0 \text { if } \beta>1 ; \quad \lim _{A \rightarrow 0^{+}} \varphi(\beta, A)=0 \text { if } \beta \leq 1 .
$$

Hence, if $\max _{i} \beta_{i}>1, \lim _{A \rightarrow 0^{+}} Q\left(\beta_{1}, \ldots, \beta_{n}, A\right)>0$ and we conclude that there exists a unique $A^{*}>0$ such that $Q\left(\beta_{1}, \ldots, \beta_{n}, A^{*}\right)=A^{*}$.

If $\max _{i} \beta_{i} \leq 1, \lim _{A \rightarrow 0^{+}} Q\left(\beta_{1}, \ldots, \beta_{n}, A\right)=0$, and one needs to compute its derivative at $A=0$. Using (15), one has

$$
\left.\frac{d}{d A} Q\left(\beta_{1}, \ldots, \beta_{n}, A\right)\right|_{A=0}=\frac{\beta_{g}}{n} \sum_{j=1}^{n} \frac{1}{1-\beta_{j}} .
$$


If the expression in (19) is greater than 1 (including the case of $\max _{i} \beta_{i}=1$ where formally it is infinite), there exists a unique $A^{*}$ such that $Q\left(\beta_{1}, \ldots, \beta_{n}, A^{*}\right)=A^{*}$.

Otherwise $Q\left(\beta_{1}, \ldots, \beta_{n}, A\right)<A$ for all $A>0$. The three possible cases for the function $Q$ are depicted in Figure 1 .

Notice that we had shown before that $R_{0}>1$ if either $\max _{i} \beta_{i} \geq 1$, or the expression in (19) is greater than 1 . Thus, a solution $A^{*}$ exists if and only if $R_{0}>1$, a special case of the result proved in [3].

For further use, note that, when a solution $A^{*}>0$ exists,

$$
Q\left(\beta_{1}, \ldots, \beta_{n}, A\right)>[<] A \Longleftrightarrow A<[>] A^{*} .
$$

Proof (of Theorem 3) First of all, note that the assumptions of the Theorem imply $\bar{\beta}+\beta_{g}>1$. Then, there exists a solution $\bar{z}$ of (11) with $\bar{z}>0$.

We now prove that, under the assumptions of the theorem, $\frac{\partial^{2} \varphi}{\partial \beta^{2}}(\beta, \bar{z})<0$ for $\beta_{\min } \leq \beta \leq \beta_{\max }$.

Condition a) obviously implies a') in the assumptions of Lemma 2.

As for case b), by looking at the proof of Lemma 2 , one notices that $\frac{\partial^{2} \varphi}{\partial \beta^{2}}(\beta, \bar{z})<0$ holds if, for all $\beta \geq \beta_{\min }, g(\beta, \varphi(\beta, \bar{z}))<0$ with $g$ defined in that proof. As $g(\beta, x)<0$ for all $x$ if $\beta>9 / 8$, the claim holds if one can prove that, for all $\beta \in\left[\beta_{\min }, 9 / 8\right]$, $\varphi(\beta, \bar{z})<x_{-}(\beta)$, the smaller root of $g(\beta, x)=0$, existing for $\beta \leq 9 / 8$. By choosing $\beta_{g}$ sufficiently small, $\varphi(\beta, \bar{z})$ can be made arbitrarily close to $\varphi\left(\beta, 0^{+}\right)$; hence, it is enough proving that $\varphi\left(\beta, 0^{+}\right)<x_{-}(\beta)$ for all $\beta$ such that $1<\beta \leq 9 / 8$.

Since $\varphi\left(\beta, 0^{+}\right)$is the positive root of $F(z, \beta, 0)$ (Lemma 1) with $F$ concave, $\varphi\left(\beta, 0^{+}\right)<$ $x_{-}(\beta)$ is equivalent to $F\left(x_{-}(\beta), \beta, 0^{+}\right)<0$. Now

$$
x_{-}(\beta)=1-\frac{3}{4 \beta}-\frac{\sqrt{9-8 \beta}}{4 \beta}
$$

and

$$
\begin{gathered}
F\left(x_{-}(\beta), \beta, 0\right)=\beta\left(1-\frac{3}{4 \beta}-\frac{\sqrt{9-8 \beta}}{4 \beta}\right)+\log \left(\frac{3}{4 \beta}+\frac{\sqrt{9-8 \beta}}{4 \beta}\right) \\
=\beta-\frac{\sqrt{9-8 \beta}}{4}+\log (3+\sqrt{9-8 \beta})-\log (\beta)-\frac{3}{4}-\log (4) \\
=\beta-\frac{\sqrt{9-8 \beta}}{4}-\log (3-\sqrt{9-8 \beta})+\log (2)-\frac{3}{4}
\end{gathered}
$$

Simple computations show that the last expression computed in $\beta=1$ is equal to 0 , and that its derivative with respect to $\beta$ is negative. Hence for all $\beta \in(0,9 / 8]$, $F\left(x_{-}(\beta), \beta, 0\right)<0$, showing that $\varphi\left(\beta, 0^{+}\right)<x_{-}(\beta)$. This proves that $\left.\mathrm{b}^{\prime}\right)$ of Lemma 2 holds for all $\beta$.

Finally, using c) in (11) yields

$$
G(2 / 3)=-\log (3)+\frac{2}{3}\left(\bar{\beta}+\beta_{g}\right) \geq-\log (3)+\frac{2}{3}\left(\beta_{\min }+\beta_{g}\right) \geq 0
$$


hence $\bar{z} \geq 2 / 3$.

Now

$F(2 / 3, \beta, \bar{z}) \geq F(2 / 3, \beta, 2 / 3)=-\log (3)+\frac{2}{3}\left(\beta+\beta_{g}\right) \geq-\log (3)+\frac{2}{3}\left(\beta_{\min }+\beta_{g}\right) \geq 0$

implying $\varphi(\beta, \bar{z}) \geq 2 / 3$, i.e. $\left.\mathrm{c}^{\prime}\right)$.

As either a'), b') or c') is satisfied, Lemma 2 shows that $\frac{\partial^{2} \varphi}{\partial \beta^{2}}(\beta, \bar{z})<0$ for all $\beta \geq \beta_{\min }$. We can then apply Jensen inequality to the function $\varphi(\cdot, \bar{z})$ and obtain

$$
\frac{1}{n} \sum_{i=1}^{n} \varphi\left(\beta_{i}, \bar{z}\right) \leq \varphi\left(\frac{1}{n} \sum_{i=1}^{n} \beta_{i}, \bar{z}\right)=\varphi(\bar{\beta}, \bar{z})
$$

where $\bar{\beta}=\frac{1}{n} \sum_{i=1}^{n} \beta_{i}$ is the average value of the $\beta_{i}$ s.

Therefore

$$
Q\left(\beta_{1}, \ldots, \beta_{n}, \bar{z}\right)=\frac{1}{n} \sum_{i=1}^{n} \varphi\left(\beta_{i}, \bar{z}\right) \leq \varphi(\bar{\beta}, \bar{z})
$$

with a strict inequality, unless $\beta_{i} \equiv \bar{\beta}$, the case discussed in the previous Theorem. As $\bar{z}$ solves (11), it follows that $\bar{z}=\varphi(\bar{\beta}, \bar{z})$.

Hence, (21) implies $Q\left(\beta_{1}, \ldots, \beta_{n}, \bar{z}\right)<\bar{z}$. It follows from (20) that $A^{*}<\bar{z}$.

Finally, by the definition of the function $Q, A^{*}=Q\left(A^{*}\right)=\frac{1}{n} \sum_{j=1}^{n} \varphi\left(\beta_{j}, A^{*}\right)$ is the average attack ratio in the groups and is lower than the attack ratio $\bar{z}$ that would have been obtained with $\beta_{i} \equiv \bar{\beta}$.

Remark 1 It is interesting to note that Theorem 3 shows that, under the assumptions of the Theorem, the average attack ratios in the groups is lower than the attack ratio caused by the average $\beta$. The same does not necessarily hold for the averages weighted by population size:

$$
\overline{z_{w}}=\frac{\sum_{i=1}^{n} N_{i} \varphi\left(\beta_{i}, A^{*}\right)}{\sum_{i=1}^{n} N_{i}} \text { and } \overline{\beta_{w}}=\frac{\sum_{i=1}^{n} N_{i} \beta_{i}}{\sum_{i=1}^{n} N_{i}} .
$$

On the other hand, if expression (3) holds for $\lambda_{g}$, repeating (with obvious modifications) the proof of Theorem 3 leads to the conclusion that the average attack ratio $\overline{z_{w}}$ is lower than the attack ratio obtained with all $\beta_{i} \equiv \overline{\beta_{w}}$.

\subsection{Numerical results}

The thesis of Theorem 3 has been proved under alternative conditions, of which the simplest one is that $\beta_{i}>9 / 8$ for all $i=1 \ldots n$. The assumptions of the Theorem have been used in order to be able to apply Jensen's inequality, a key step that leads to 
the result. Indeed, the exact numbers appearing in the assumptions are derived from purely technical arguments, and do not seem to have any clear biological interpretation. It is possible that, using a different approach, weaker conditions could be found that ensure that the average attack ratio is lower in the heterogeneous model than in the homogeneous model.

However, it is clear that some conditions are necessary to ensure the result. Indeed, in the extreme case where $\bar{\beta}+\beta_{g} \leq 1$ but some $\beta_{i}>1$, there would be no epidemic (hence 0 attack ratio) in the homogeneous model, while even a single $\beta_{i}>1$ ensures that $R_{0}>1$ in the heterogeneous model, and thus a positive attack ratio would result. In general, when $\bar{\beta}$ is low, we expect that the attack ratio would be larger in the heterogeneous model than in the homogeneous one.

This issue has been explored through numerical computations in the simplest case, namely $n=2$. In Fig. 2 we show how the attack ratio depends on the contact rates $\beta_{1}$ and $\beta_{2}$ in the two groups. As anticipated, for large values of $\beta_{i}$ attack ratios are higher in the homogeneous model, while for lower values of $\beta_{i}$, the situation is reversed. When $\beta_{g}$ is large (panel d), the threshold between the two regions seems to depend on the value of $\bar{\beta}$ only, while for $\beta_{g}$ closer to 0 , the boundary has a less trivial shape.

\section{Comparison with stochastic simulations}

As mentioned in the Introduction, a reason for considering models like (1) is the acknowledgment that the dynamics of actual epidemics can be captured only in models that allow for population mixing at different levels.

As the detailed mixing structure may be however hard to ascertain, one may ask whether aggregate estimates such as the reproduction ratio $R_{0}$ can be useful to gauge the predicted impact of an emerging epidemic.

The immediate answer arising from the analysis in the previous Section is negative: if within-group transmission rates are heterogeneous in the different groups, $R_{0}$ will be higher than with homogeneous transmission rates, while Theorem 3 shows that, under many assumptions on the parameters, the average attack ratio will be lower. Furthermore, equations (7) and (5) show that the numerical difference in the value of $R_{0}$ may be large, and so can be the difference in attack ratios (see panel (a) of Fig. 2). Thus, the relation between the value of $R_{0}$ and final attack ratio $z$ holding for the simple SIR model $[30,17]$, assuming that $\gamma$ is known, breaks down for model (1) when within-group transmission rates are heterogeneous.

Note however that, in actual epidemics, one generally does not attempt to estimate the true value of $R_{0}$ by solving equation (6) or something similar. Rather, the exponential growth rate $r$ is estimated from aggregated data, and is then converted into an estimate of $R_{0}$ through a theoretical relation between the two quantities [42,36]. One may then be interested in studying the relation between this estimate and final attack ratio.

Moreover, it is clear that (1) represents only an approximation of more complex transmission structures, and that stochastic events may play a relevant role in infection 


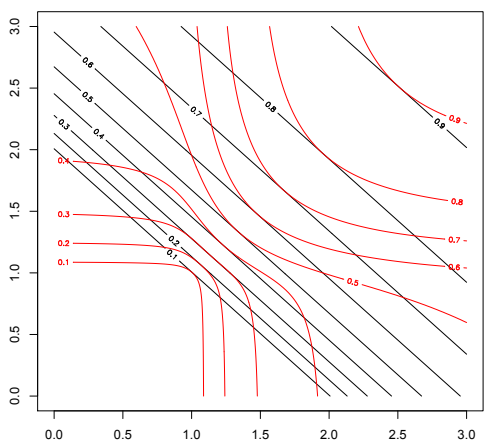

(a)

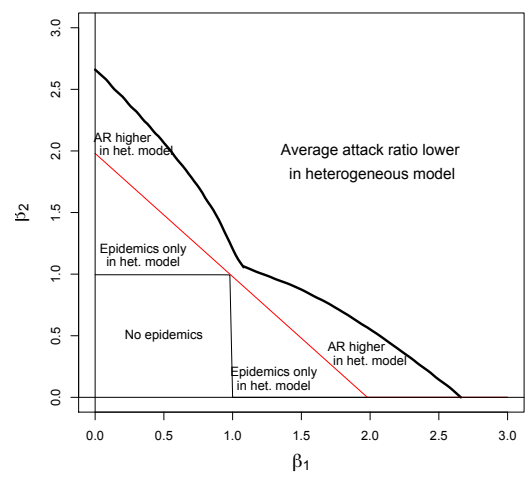

(c)

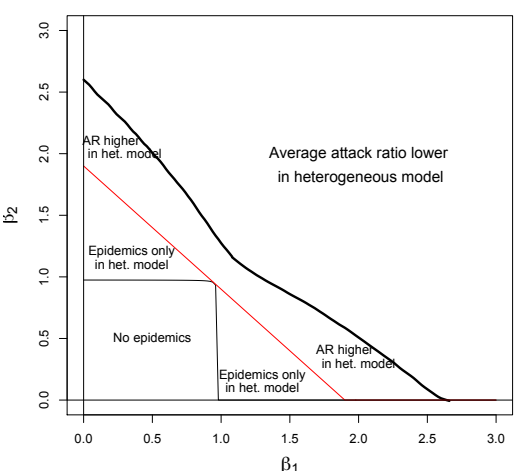

(b)

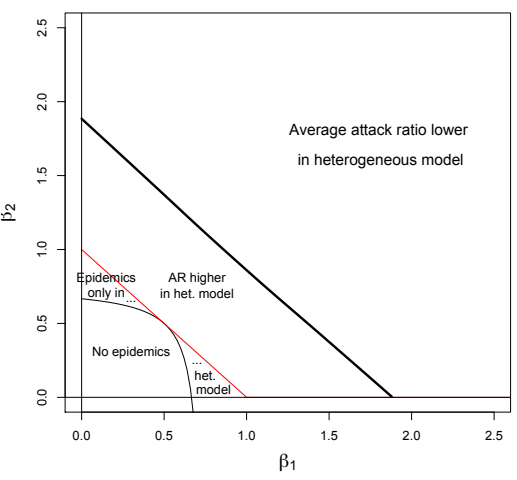

(d)

Fig. 2: Comparison between average attack ratios in the models with heterogeneous or homogeneous transmission, depending on the parameters $\beta_{1}$ and $\beta_{2}$. (a) Lines of constant average attack ratio in the parameter plane $\left(\beta_{1}, \beta_{2}\right)$; in black those for the homogeneous model with $\bar{\beta}=\left(\beta_{1}+\beta_{2}\right) / 2$ (hence straight lines), in red those for the heterogeneous model. Value of $\beta_{g}=0.05$. (b) Using the values from panel (a), subdivision of the parameter space into a region where attack ratio is lower with heterogeneous transmission, one in which the opposite happens, one in which $R_{0}>1$ only with heterogeneous transmission, and finally one ('No epidemics') with $R_{0}<1$ for both types of transmission. (c) like (b) with $\beta_{g}=0.01$. (d) like (b) with $\beta_{g}=0.5$. 
transmission, especially if groups are small.

There are then two questions that we wish to address:

- does the solution of equation (10) approximate the final attack ratio obtained in stochastic simulations, so that the conclusions of Theorem 3 hold also in a stochastic setting?

- If $R_{0}$ is estimated from aggregated data through an estimate of the exponential growth rate, would it have a relation with average attack ratio similar to that holding for the homogenous model?

We examine these questions through simulations tailored for groups of intermediate size (such as schools, for instance, or informal community groups) for which it is plausible that within-group transmission can be described through differential equations.

Precisely, stochasticity has been modelled as a continuous-time Markov chain whose rates have been specified consistently with the rates of the SIR deterministic model. The algorithm for implementing the Markov chain follows the so-called Gillespie algorithm [20] that was actually already proposed in [29]; it consists of the repetition of three steps that update the state of the population and time (see also [40]):

1. Update time: the time interval between two subsequent events is exponentially distributed with average $T^{-1}$ with

$$
T=\sum_{i=1}^{n} \sum_{i=1}^{n} \gamma I_{i}+\sum_{i=1}^{n} \beta_{i} S_{i} \frac{I_{i}}{N_{i}}+\sum_{i=1}^{n} \frac{\beta_{g}}{n} S_{i} \sum_{j=1}^{n} \frac{I_{j}}{N_{j}} ;
$$

2. Randomly select an event which can be the infection of a susceptible individual (with probability $\left(\sum_{i} \beta_{i} S_{i} \frac{I_{i}}{N_{i}}+\sum_{i} \frac{\beta_{g}}{n} S_{i} \sum_{j} \frac{I_{j}}{N_{j}}\right) / T$ or the removal of an infected individual (with probability $\sum_{i} \gamma I_{i} / T$ );

3. Update the state of the population according to the event occurred.

As reference values for the simulations considered here, we took the case where in a homogeneous model, $R_{0}$ would be between 1.4 and 1.8 , close to the values considered for influenza $[22,15,13]$, and varied the levels of heterogeneity and of connection between groups. Precisely, we used $R=1.4,1.6$ and 1.8; set global transmission rate to $\beta_{g}=\varepsilon R$ with either $\varepsilon=0.03125$ or $\varepsilon=0.3125$. For each such value, we randomly sampled the values of $\beta_{i}$ from a log-normal distribution with mean $R(1-\varepsilon)$ (so that according to (5) $R_{0}=\bar{\beta}+\beta_{g} \approx R$ ) and variance either 0 (homogeneous case), 0.5 or 2.5.

For each scenario, we repeated 10 times the random sample of transmission rates $\beta_{i}$; for each (random) choice of parameters, we ran a number of stochastic simulations, seeded by 1 infectious individual, randomly placed in one of the groups. As in all stochastic models, disease fadeout can happen after the first few cases; we defined 'major' epidemics the simulations resulting in at least $10 \%$ of the total population having been infected. While the threshold is somewhat arbitrary, it is well known that, if population size is large enough, the number of cases generated by a stochastic epidemic has a bimodal distribution [2] and indeed almost all simulations ended up 


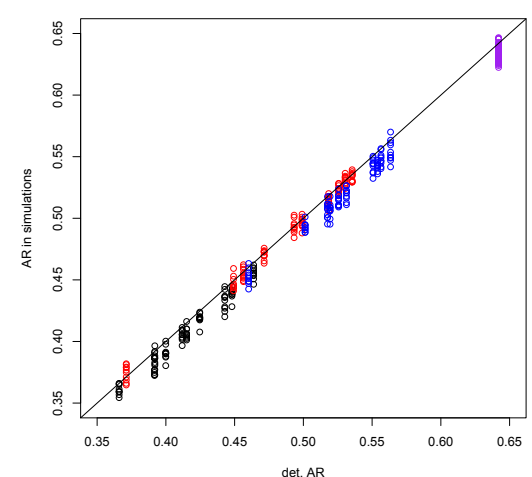

(a)

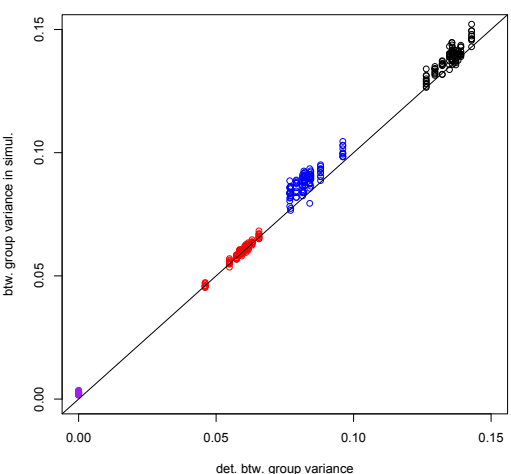

(b)

Fig. 3: (a) On the $x$-axis average attack ratios in the deterministic model (1) for different parameter values; on the $y$-axis average attack ratios of stochastic simulations with the same parameter values. In black, values obtained with $\varepsilon=0.03125$ and $\beta_{i}$ sampled from a lognormal with mean $1.6(1-\varepsilon)$ and variance 2.5 ; in red, same except that $\varepsilon=0.3125$; blue, same as black but variance of lognormal equal to 0.5 ; purple, same as black but variance of the lognormal equal to 0 . (b) On the $x$-axis variance (among groups) of attack ratios in the deterministic model (1); on the y-axis variance (among groups) of attack ratios in stochastic simulations. Parameters and legend as in (a).

very far from the threshold. Thus, for each parameter sample, we ran enough simulations to obtain 10 'major' epidemics. We varied also the number of groups and their size, but in what follows we show only the case with $n=100$ and $N_{i} \equiv 1,000$, thus with a total population size of 100,000 .

A comparison between the final attack ratio in the deterministic model (1) and stochastic simulations is shown in Fig. 3. It can be seen that the correspondence is very good: different parameter samples yield different values for the predicted attack ratio in model (1) and for the variance in attack ratios between groups. The results of the stochastic simulations are all very close to the deterministic predictions. Comparing black, blue and purple dots in panel a) of Fig. 3, one can also see that overall attack ratio tends to decrease with increasing variance in the within-group transmission rates, as long as other parameters are kept constant.

It must be remembered that only stochastic simulations leading to a 'major' epidemic are shown, while many others result in early extinction of the epidemic. The probability of early extinction in homogeneous models is equal to $1-1 / R_{0}$ [2], but in heterogenous models it is necessary to take into account the properties of the group in which the first introduction occurs $[10,33,14]$, and there does not seem to be a simple formula for the case considered here. Relationships of the probability of ex- 
tinction in more complex stochastic model with several possible predictors have been extensively explored in [16].

In principle, the initial growth rate of an epidemic $r$ could be obtained by linearizing (1) near $(\mathbf{N}, \mathbf{0})$ and computing the largest eigenvalue of the resulting matrix, i.e. $K^{t}-I$ with $K$ defined in (4) (remember that $\gamma=1$ ); hence, we would obtain $r=R_{0}-1$. However, this derivation neglects the fact that, with finite population, the infection will initially be present in a single group, and then will spread (faster or slower depending on the strength of global transmission) to other groups. If group transmission rates differ significantly among each other, $R_{0}$ will be dominated by the group with highest transmission, while patterns of epidemic growth will depend on transmission rates within many groups, and on the speed of transmission between groups.

Hence, from individual simulations we estimated the exponential growth rate $r$ in an empirical way, mimicking what is often performed on actual data [31]. Namely, we fitted a line to the logarithms of simulated incidence values over different intervals in their initial growth part, keeping only the fits over intervals that included at least 4 points and yielded an $R^{2}$ of the regression above $95 \%$. Finally, we averaged the estimated slopes to yield an estimate of $r$ for that simulation. In Fig. 4(a) we show the

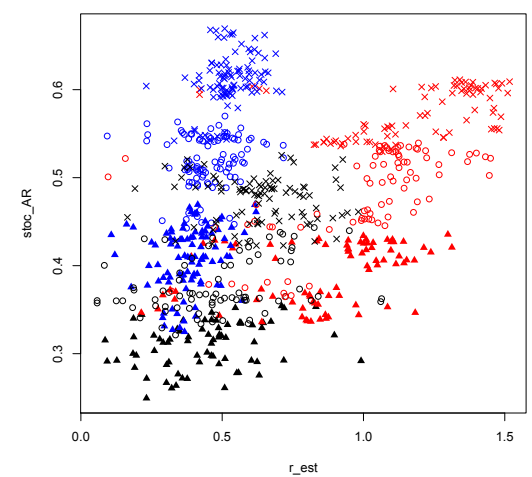

(a)

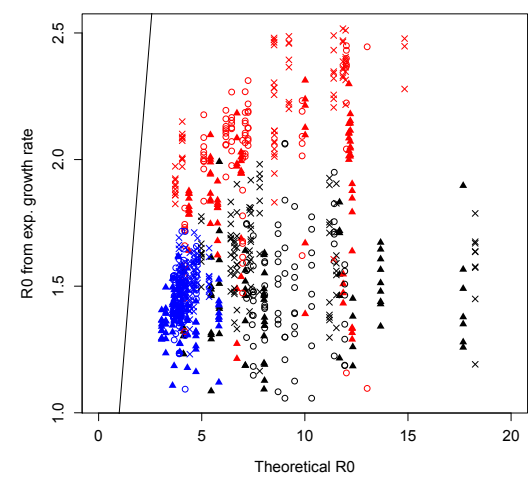

(b)

Fig. 4: (a) Estimated exponential growth rate $r$ ( $x$-axis) vs. average attack ratio (yaxis) in the different simulations obtained with $\beta_{g}=R \varepsilon$ and $\beta_{i}$ sampled from a lognormal with mean $R(1-\varepsilon)$ and variance $\sigma^{2}$. Solid triangles correspond to $R=1.4$, open circles to $R=1.6$, crosses to $R=1.8$; in black, points obtained with $\varepsilon=0.03125$ and $\sigma^{2}=2.5$; in red, $\varepsilon=0.3125$ and $\sigma^{2}=2.5$; blue, $\varepsilon=0.03125$ and $\sigma^{2}=0.5$. (b) $R_{0}$ from formula (6) ( $x$-axis) vs. $R_{0}=1+r$, using the estimated $r$ in the different simulations. Point symbols as in (a); the solid line is the bisectrix $R_{0, \text { est. }}=R_{0 \text {,theor. }}$

estimates of $r$ compared to the attack ratios in the same simulations. It can be seen that both $r$ and the attack ratios increase with $R$; moreover the estimated exponential 
growth rate is higher when groups are more connected $(\varepsilon=0.3125$, red points in Figure (4)). On the other hand, for a given estimated $r$, attack ratio tend to be higher when between-group variance in transmission rate is lower (blue points in Figure (4)). Overall, one can see a (statistically significant) relation between the estimates of $r$ and final attack ratios, even if one restricts the analysis to different samples of $\beta_{i}$ within the same simulation scenario. However, the relationships strongly depend on the simulations included in the analysis; thus, it appears to be difficult to infer a general rule connecting estimated exponential growth rate and epidemic impact.

We finally compare (Fig. 4(b)) the values of $R_{0}$ obtained from the theoretical formula (6) to those obtained from the estimated exponential growth rate using the formula $R_{0} \approx 1+r T_{I}$ [42]. It can be seen that the values of $R_{0}$ obtained from (6) are extremely large, especially when transmission rates $\beta_{i}$ are sampled from the lognormal with higher variance and groups are weakly connected (black points in Figure 4, where a point with $R_{0} \approx 35$ is not shown) and are very weakly correlated to the values of $R_{0}$ obtained from the estimated values of $r$. Hence, while $R_{0}$ correctly determines the reduction in transmission rate necessary for bringing an epidemic below threshold, the epidemic dynamics is definitely very different from that of a homogeneous model with that value of $R_{0}$. Note that in the simulations we used $n=100$ groups; if the value of $n$ were smaller, or the variance in contact rates were smaller, the values of the theoretical $R_{0}$ would presumably be smaller.

We remark that different concepts of $R_{0}$, each catching a relevant aspect of the transmission process and potentially more useful for populations stratified into (relatively small) groups with different mixing patterns, have been proposed and analysed [21, $38,8]$. We are not discussing these here.

To summarise, we found that the final attack ratio predicted from the deterministic model (1) is an extremely good predictor of the outcome of stochastic simulations that survive early extinction, at least when groups are rather large, as it happens in our simulations. On the other hand, even in the simple context of the models examined here, although there is a correlation between the estimated exponential growth rate and final attack ratio, there does not seem to be a simple relationship between them, mimicking the one existing in homogeneous models.

Several properties of the model (for instance, the level of connection among groups; the variance in transmission rates) affect the final attack ratio, beyond what can be gathered from initial growth. An open question, to which the present work may give at best preliminary insights, is whether some summary measures of contact structure can be built that help in predicting final attack rates. Presumably, the analytical approximations obtained for effective early exponential growth rate [39] or final attack ratio [27] could help towards that. However, it has also be remembered that final attack rates predicted by metapopulation models (like the ones examined here) can be rather different from those obtained by corresponding agent-based models where individual contacts are kept track of [1].

Acknowledgments

We thank two anonymous reviewers, whose comments have greatly improved the manuscript. 


\section{References}

1. M. Ajelli, B. Gonçalves, D. Balcan, V. Colizza, H. Hu, J. Ramasco, S. Merler, and A. Vespignani. Comparing large-scale computational approaches to epidemic modeling: Agent-based versus structured metapopulation models. BMC Infect. Dis., 10(1):190, 2010.

2. H. Andersson and T. Britton. Stochastic Epidemic Models and Their Statistical Analysis. Springer, 2000.

3. V. Andreasen. The final size of an epidemic and its relation to the basic reproduction number. Bull. Math. Biol., 73(10):2305-2321, 2011.

4. V. A. Andreasen and F. B. Christiansen. Persistence of an infectious disease in a subdivided population. Math. Biosc, 96:239-253, 1989.

5. F. Ball. Deterministic and stochastic epidemics with several kinds of susceptibles. Adv. Appl. Probab., 17(01):1-22, 1985.

6. F. Ball, T. Britton, T. House, V. Isham, D. Mollison, L. Pellis, and G. Scalia Tomba. Seven challenges for metapopulation models of epidemics, including households models. Epidemics, 10:63-67, 2015.

7. F. Ball, D. Mollison, and G. Scalia-Tomba. Epidemics with two levels of mixing. Ann. Appl. Probab., 7:46-89, 1997.

8. F. Ball, L. Pellis, and P. Trapman. Reproduction numbers for epidemic models with households and other social structures II: Comparisons and implications for vaccination. Math. Biosci., 274:108-139, 2016.

9. F. Ball, D. Sirl, and P. Trapman. Analysis of a stochastic SIR epidemic on a random network incorporating household structure. Math. Biosci., 224(2):53-73, 2010.

10. N. Becker and I. Marschner. The effect of heterogeneity on the spread of disease. In J.-P. Gabriel, C. Lefèvre, and P. Picard, editors, Stochastic Processes in Epidemic Theory, Proc. Conf. Luminy, Oct. 23-29, 1988, pages 90-103. Springer, Berlin Heidelberg 1990.

11. L. Billard. A Stochastic General Epidemic in m Sub-Populations. J. Appl. Probab., 13(3):567-572, 1976.

12. S. Blythe and C. Castillo-Chavez. Like-with-like preference and sexual mixing models. Math. Biosci., , 96:221-238, 1989.

13. P.-Y. Boëlle, S. Ansart, A. Cori, and A.-J. Valleron. Transmission parameters of the A/H1N1 (2009) influenza virus pandemic: a review. Influenza Other Respi. Viruses, 5(5):306-316, 2011.

14. L. Bolzoni, A. Pugliese, and R. Rosà. The role of heterogeneity on the invasion probability of mosquito-borne diseases in multi-host models. J. Theor. Biol., 377:25-35, 2015.

15. G. Chowell, M. A. Miller, and C. Viboud. Seasonal influenza in the United States, France, and Australia: transmission and prospects for control. Epidemiol. Infect., 136(06):852-864, 2008.

16. P. C. Cross, P. L. F. Johnson, J. O. Lloyd-Smith, and W. M. Getz. Utility of R0 as a predictor of disease invasion in structured populations. J. R. Soc. Interface, 4(13):315-324, 2007.

17. O. Diekmann and J. A. P. Heesterbeek. Mathematical Epidemiology of Infectious Diseases. Wiley, Chichester, New York, 2000.

18. S. N. Ethier and T. G. Kurtz. Markov Processes. Characterization and Convergence. John Wiley \& Sons, 1986.

19. N. M. Ferguson, D. A. T. Cummings, S. Cauchemez, C. Fraser, S. Riley, A. Meeyai, S. Iamsirithaworn, and D. S. Burke. Strategies for containing an emerging influenza pandemic in Southeast Asia. Nature, 437(7056):209-214, 2005.

20. D. T. Gillespie. Exact stochastic simulation of coupled chemical reactions. J. Phys. Chem., 81(25):2340-2361, 1977.

21. E. Goldstein, K. Paur, C. Fraser, E. Kenah, J. Wallinga, and M. Lipsitch. Reproductive numbers, epidemic spread and control in a community of households. Math. Biosci., 221(1):11-25, 2009.

22. R. F. Grais, J. H. Ellis, A. Kress, and G. E. Glass. Modeling the spread of annual influenza epidemics in the \{U.S.\}: the potential role of air travel. Health Care Manag. Sci., 7:127-134, 2004.

23. T. J. Hagenaars, C. A. Donnelly, and N. M. Ferguson. Spatial heterogeneity and the persistence of infectious diseases. J. Theor. Biol., 229(3):349-359, 2004.

24. M. E. Halloran, N. M. Ferguson, S. Eubank, I. M. Longini, D. A. T. Cummings, B. Lewis, S. Xu, C. Fraser, A. Vullikanti, T. C. Germann, D. Wagener, R. Beckman, K. Kadau, Macken, D. S. Burke, and P. Cooley. Modeling targeted layered containment of an influenza pandemic in the United States. Proc Natl Acad Sci USA, 105:4639-4644, 2008.

25. H. W. H. Hethcote and J. A. Yorke. Gonorrhea Transmission Dynamics and Control. Springer, 1984. 
26. T. House and M. J. Keeling. Deterministic epidemic models with explicit household structure. Math. Biosci., 213:29-39, 2008.

27. T. House, J. V. Ross, and D. Sirl. How big is an outbreak likely to be? Methods for epidemic final-size calculation. Proc. R. Soc. London A, 469(December 2012):20120436, 2013.

28. M. J. Keeling and C. A. Gilligan. Bubonic plague: a metapopulation model of a zoonosis. Proc. $R$ Soc. London B, 267:2219-2230, 2000.

29. D. G. Kendall. An artificial realization of a simple "Birth-and-Death" process. J. R. Stat. Soc. Ser. B, 12(1):116-119, 1950.

30. W. O. Kermack and A. G. McKendrick. A contributions to the mathematical theory of epidemics. Proc. R. Soc. London A, 115:700-721, 1927.

31. A. A. King, M. de Cellès, F. M. G. Magpantay, and P. Rohani. Avoidable errors in the modelling of outbreaks of emerging pathogens, with special reference to Ebola. Proc. R. Soc. London B, 282(1806):20150347, 2015.

32. M. Lindholm and T. Britton. Endemic persistence or disease extinction: The effect of separation into sub-communities. Theor. Popul. Biol., 72(2):253-263, 2007.

33. A. L. Lloyd, J. Zhang, and A. M. Root. Stochasticity and heterogeneity in host-vector models. J. R. Soc. Interface, 4(16):851-863, 2007.

34. R. M. May, R. M. Anderson, and M. E. Irwin. The transmission dynamics of Human Immunodeficiency Virus (HIV) [and Discussion]. Philos. Trans. R. Soc. London. B, 321(1207):565-607, 1988.

35. S. Merler and M. Ajelli. The role of population heterogeneity and human mobility in the spread of pandemic influenza. Proc. R. Soc. London B, 77:557-565, 2010.

36. H. Nishiura, G. Chowell, M. Safan, and C. Castillo-Chavez. Pros and cons of estimating the reproduction number from early epidemic growth rate of influenza A (H1N1) 2009. Theor. Biol. Med. Model., 7(1), 2010.

37. A. Nold. Heterogeneity in disease-transmission modeling. Math. Biosci., 52:227-240, 1980.

38. L. Pellis, F. Ball, and P. Trapman. Reproduction numbers for epidemic models with households and other social structures. I. Definition and calculation of R0. Math. Biosci., 235(1):85-97, 2012.

39. L. Pellis, N. M. Ferguson, and C. Fraser. Threshold parameters for a model of epidemic spread among households and workplaces. J. R. Soc. Interface, 6(40):979-987, 2009.

40. E. Renshaw. Stochastic Population Processes. Oxford Univ. Press, Oxford, 2011.

41. L. Sattenspiel and K. Dietz. A structured epidemic model incorporating geographic mobility among regions. Math. Biosci., 128:71-91, 1995.

42. J. Wallinga and M. Lipsitch. How generation intervals shape the relationship between growth rates and reproductive numbers. Proc. R. Soc. London B, 274(1609):599-604, 2007. 